\title{
Plasma Vortices in Planetary Wakes
}

\author{
H. Pérez-de-Tejada, Rickard Lundin and \\ D. S. Intriligator \\ Additional information is available at the end of the chapter \\ http://dx.doi.org/10.5772/52998
}

\section{Introduction}

Measurements conducted in interplanetary space and in the vicinity of planets of the solar system have shown plasma structures produced by the solar wind that resemble fluid dynamic features; namely, vortex rotations within the earth's magnetosphere and also along the Venus wake. In both planets the solar wind encounters different obstacles since the earth is protected by its intrinsic magnetic field that is compressed by the dynamic pressure of the solar wind to form a large size cavity (the magnetosphere) that bounds its direct approach to the earth's vicinity. At Venus the conditions are different since the planet does not have an internal magnetization that would produce an earth-type magnetic obstacle to the solar wind. Instead, the latter reaches directly upon the upper layers of the planet's atmosphere and interacts with its ionized components (the ionosphere). The outcome of this interaction is a plasma wake of large extent whose geometry is similar to that of the earth's magnetospheric tail but that arises from conditions that are different in both planets. While there is evidence for the observation of fluid-like vortices as the solar wind streams along the wake of the earth and Venus there is a major issue as to the manner in which they are produced. In fact, since the solar wind is a collisionless plasma; namely its charged particles barely execute collisions among them (their mean free path is comparable to one astronomical unit) it should not be expected that it behaves as a continuum when it interacts with planetary obstacles. The opposite has been verified by a variety of observations with indications that the physical properties of both the solar wind fluxes and the planetary particles that are being eroded through their interaction can be described in terms of fluid dynamic processes (a review of this issue was presented by Pérez-de-Tejada, 2012).

The motion of the solar wind particles as they interact with the earth's magnetic field is described in terms of gyromagnetic trajectories as they move across the magnetic field lines. 
Their circular (Larmor) motion is influenced by local drift displacements that are produced by space gradients of the magnetic field intensity and that carry them along the boundary of the magnetosphere. Such process also occurs while there are local electric currents produced by the different drift of the positive (mostly proton) and negative (electron) components of the solar wind. Throughout the front part (dayside) of the magnetosphere the motion of those particles is directed by magnetic (Lorentz) forces which guide them along the magnetic field lines in an environment where the local magnetic energy density is larger than their kinetic energy density (Under such conditions the transport speed of magnetic signals, i.e. the Alfven speed, is larger than the particles' speed and the flow is labeled subalfvenic). The gyrotropic motion of the solar wind particles as they encounter the earth's magnetic field provides a mechanism that leads to a continuum transport of their properties despite the fact that they do not collide with each other. Alternate conditions are encountered along the magnetospheric tail where the magnetic field intensity has decreased significantly with the downstream distance from the earth and the speed of the solar wind particles has increased to nearly its freestream values (the local flow is expected to achieve superalfvenic conditions). Data that will be addressed below show the presence of vortical plasma structures within the magnetospheric tail and that reveal effects that can be related to Kelvin-Helmholtz instabilities (Wolfe et al., 1980: Terada et al:, 2002) associated to a fluid dynamic response in their motion rather than to trajectories directed by the magnetic field.

The overall manner in which the solar wind responds as it interacts with the Venus ionosphere is different since in the absence of an intrinsic planetary magnetization its particles reach the ionospheric plasma and, at the same time, also encounter ions that are located in the outer exosphere of the planet. Thus, the solar wind particles do not enter a region dominated by an intrinsic planetary magnetic field where they would be guided to carry out gyromagnetic trajectories as it is the case in the earth's magnetosphere. Instead, the processes produced as a result of their interaction with the planetary ions are more complex since the latter ions are accelerated by a (convective) electric field that derives from the relative velocity difference that exists between them and the solar wind (the planetary oxygen ions of the Venus exosphere are subject to the effects of that electric field). The result of that process is labeled mass loading and leads the planetary ions to execute gyromagnetic trajectories as they travel through the solar wind and its convected solar magnetic field. The magnetic field intensity of the solar wind becomes enhanced around the upper boundary of the dayside ionosphere (ionopause) in a layer labeled ${ }^{\circ}$ magnetic barrier ${ }^{\circ}$ that is produced by the solar wind - ionosphere interaction. Despite the acceleration of the exospheric planetary ions by the solar wind measurements show that the bulk of the solar wind momentum is mostly transferred to the upper Venus ionosphere where it strongly contributes to produce the nightward directed trans-terminator flow inferred from the Pioneer Venus Orbiter (PVO) data that was reported by Knudsen et al., (1980).

Processes associated with this effect are different from those expected in gyrotropic trajectories since they lead to the observation of vortical plasma structures in the near Venus wake associated with superalfvenic flow conditions (the kinetic energy density of the plasma being larger than the local magnetic energy density). The origin of the continuum response of 
the solar wind as it streams around and behind Venus does not seem to be only related to gyromagnetic trajectories but to a fluid-like behavior generated by other particle motions. Strong magnetic and plasma turbulence that has been inferred from different spacecraft measurements (Bridge et al., 1967; Vörós et al., 2008) suggest that the motion of the particles may be dictated by local turbulence which should lead to a stochastic distribution of their trajectories. It is possible that wave-particle interactions may be ultimately responsible for the fluid-like character of the solar wind interaction with planetary ionospheres and the generation of plasma and magnetic vortical structures as those reported by Pope et al., (2009) from the Venus Express measurements.

\section{Plasma vortices in the earth's magnetosphere}

Measurements conducted with various spacecraft that have probed the earth's magnetospheric tail have shown that the plasma flow that streams within that region of space exhibits changes in its direction of motion that are suggestive of vortical structures. From the analysis of plasma data first obtained with the International Sun Earth Explorer (ISEE) satellites and more recently with the Cluster spacecrafts it has been possible to derive that there are regions along the tail direction of the magnetosphere where the plasma exhibits a sense of rotation (clockwise in the morning sector of the magnetosphere and counter clockwise in the evening sector both viewed from above the ecliptic plane) which suggests a wave motion that in some instances leads to vortical structures that move tailward with a speed of $300-400 \mathrm{~km} / \mathrm{s}$. The inferred features have a scale size of several earth radii nearly comparable to the width of the magnetospheric tail and it has been inferred that their rotation period is of the order of 5-20 min (Hones et al. 1978; 1981; 1983). The vortex structures form part of a wave which has a several earth radii wavelength and that increases downstream along the tail direction. From observations carried out with the Cluster spacecrafts it has also been possible to examine the plasma composition and the vortex flow dynamics at the time when there are enhanced values of the solar wind dynamic pressure (Tian et al. 2010). As a whole it is believed that vortices may derive from Kelvin-Helmholtz instabilities at the boundary of the magnetosphere (magnetopause) or at the inner edge of a plasma boundary layer within the magnetosphere.

A suitable example of the plasma and magnetic field data obtained with a Cluster spacecraft by the morning flank of the magnetosphere and far downstream from the earth (at $x=-11 R_{e}$; $y=-15 R_{e} ; z=3 R_{e} ; R_{e}$ being the earth radius) was presented by Tian et al., (2010) and is reproduced in Figure 1. Those measurements lead to the observation of a series of vortices that can be inferred from the ion velocity components marked in the lower panels of that figure. The vortex regions are indicated by the shaded bands when the $v_{x}$ component becomes large, the $\mathrm{v}_{\mathrm{y}}$ component changes sign (red profile), and the vortex rotation is derived from changes in the velocity vector orientation in the frame at the left side of the bottom panel. Comparable changes are also seen in the value of the $\mathrm{B}_{\mathrm{x}}$ and $\mathrm{B}_{\mathrm{y}}$ magnetic field components (second panel) which are derived by tracing the $\mathrm{z}$-axis along the mean magnetic field direction. 


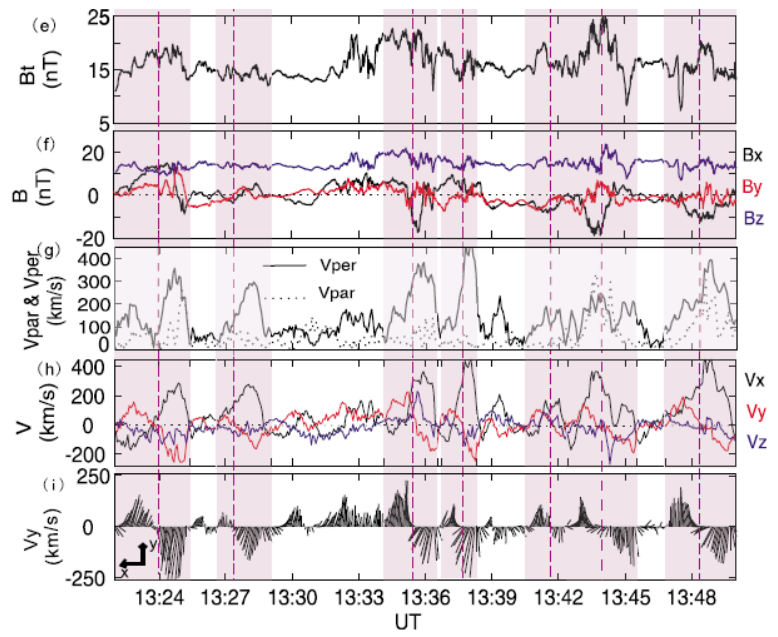

Figure 1. - A series of flow vortices in the earth's magnetosphere observed by a Cluster spacecraft on July 6, 2003. The magnetic field and its components are indicated in the top two panels and the ion velocity and its components in the two lower panels (the velocity components in a reference frame in which the $z$ and the $x$ axes are parallel and perpendicular to the average magnetic field are in the middle panel). The profiles were selected from those presented in Figure 7 of Tian et al., (2010).

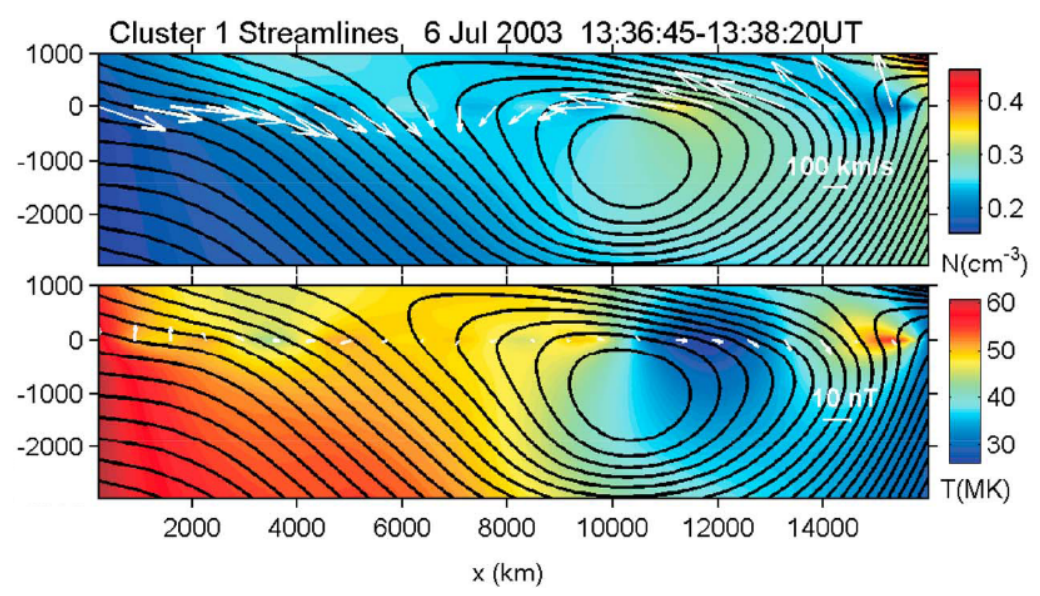

Figure 2. - Map of flow streamlines projected on the xy plane derived from the velocity rotation measured in the 13:36:45 UT - 13:38:20 UT time interval of July 6, 2003 in Figure 1. The streamlines are traced on the ion density (top panel) and on the ion temperature (lower panel) distributions (the white arrows in the top panel represent the measured velocity vector direction, and those in the lower panel the direction of the magnetic field). The streamlines describe conditions from the inner plasma sheet across the dawn side of the magnetosphere and were selected from those presented in Figure 11 of Tian et al., (2010). 
The rotation of the velocity vector implied from the lower panels in Figure 1 serves to construct streamline maps of the flow using a numerical code applied to the data points along the spacecraft trajectory. The results are reproduced in Figure 2 where the observed direction of the velocity vector projected on the xy plane is marked by the white arrows in the upper panel and the direction of the magnetic field is indicated by white arrows in the lower panel (after Tian et al., 2010). The gradual and persistent change in the direction of the velocity vector with distance in the upper panel is indicative of an anticlockwise rotating vortex structure with an approximated two dimensional 1-2 $R_{e}$ scale size. The reported observations occurred at the time when there is an enhanced solar wind dynamic pressure thus implying an intensified compression of the magnetosphere which could in this case have led to earthward moving directed vortices.

Measurements conducted with the ISEE vehicles have shown evidence of tailward directed vortical structures along the sides of the magnetosphere. However, there are indications that in the central plasma sheet of the magnetosphere the motion of the particles is observed to be earthward from the tail. An example of such measurements is reproduced in Figure 3 with evidence in the lower panel of a repeated rotation in the direction of the flow which in most cases leads to large (> $100 \mathrm{~km} / \mathrm{s}$ ) speed values (upper panel) at the time when the solar ecliptic longitude $\phi_{\mathrm{SE}}$ becomes small; i.e. when the flow is sunward directed (Hones et al., 1981).

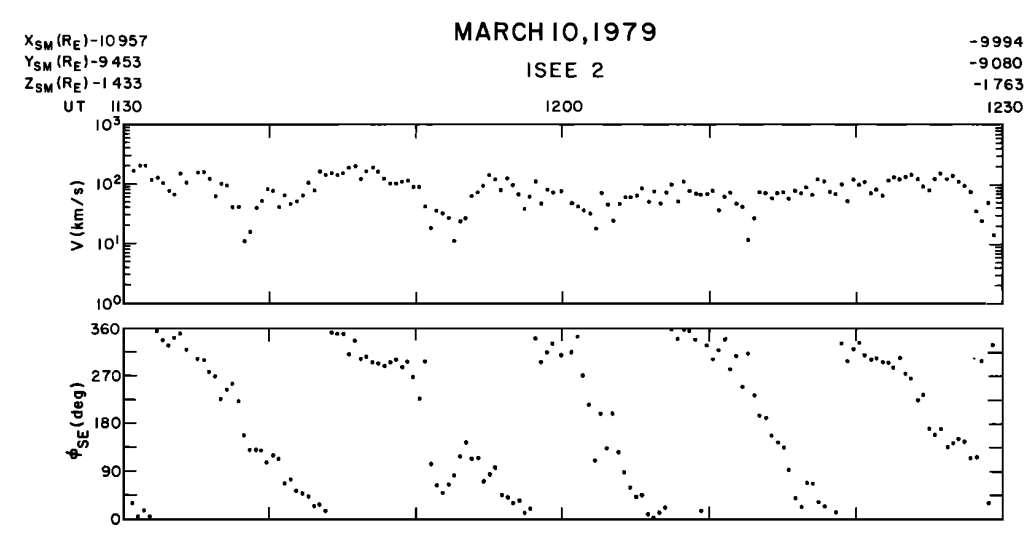

Figure 3. - Speed values (upper panel) and flow direction (lower panel) measured with the ISEE 2 spacecraft on March 10, 1979 within the earth's magnetosphere. A repeated counterclockwise rotation of the solar ecliptic longitude angle $\boldsymbol{\phi}$ is detected in the evening sector of the plasma sheet (after Hones et al., 1981).

A general schematic view of the flow distribution along the sides of the magnetosphere that has been derived from the ISEE measurements is reproduced in Figure 4. Tailward directed vortex structures occur by those regions and the diagram illustrates plasma patterns that may arise from Kelvin Helmholtz instabilities at the magnetopause and that lead to sudden changes in the flow direction when observed in the earth's rest frame. 


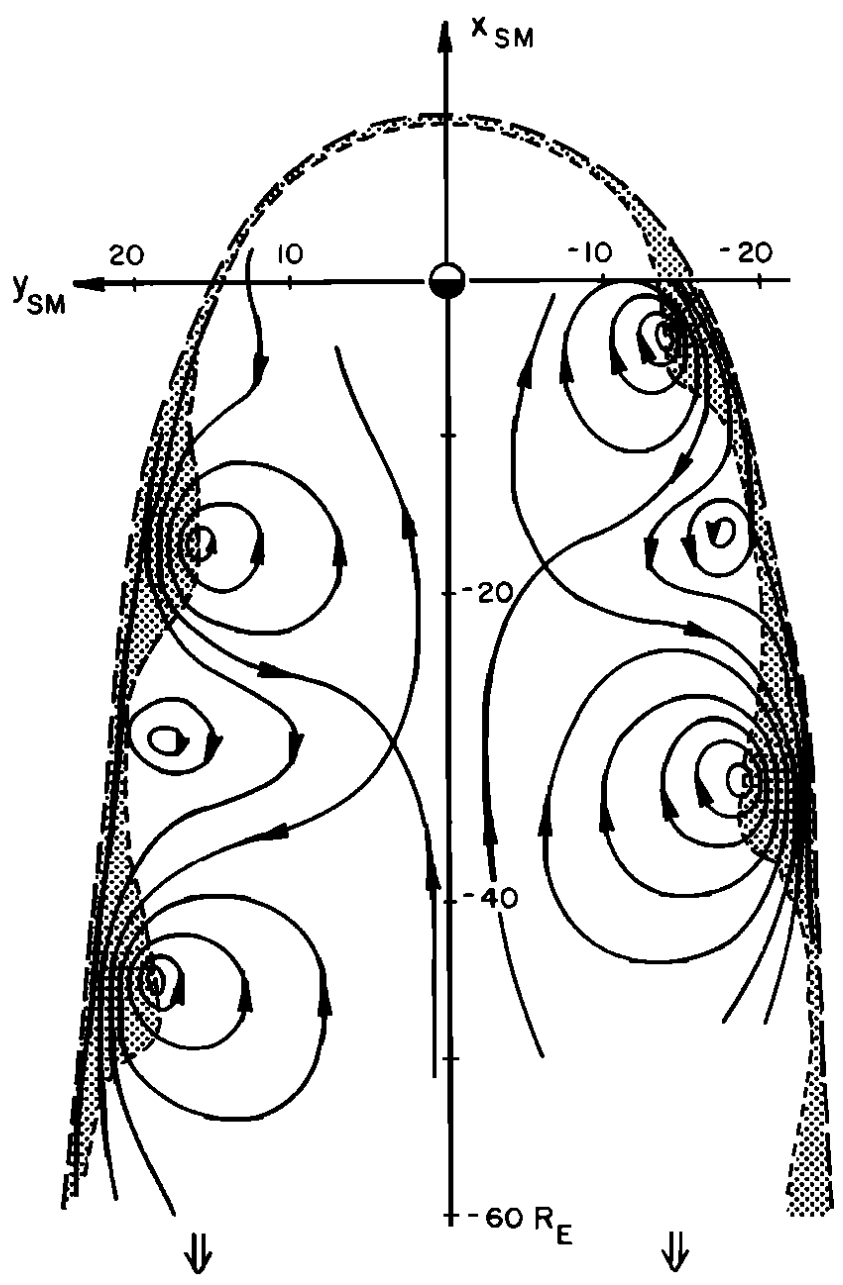

Figure 4. - Schematic description of plasma vortices within the earth's magnetosphere inferred from the ISEE measurements. The flow pattern represented by the solid lines is mostly tailward through the magnetosphere as it is indicated by the white arrows at the bottom (Hones et al., 1981).

The vortex structures are superimposed on a general circulation flow pattern within the magnetosphere in which the plasma is driven tailward along the sides by the kinetic pressure of the solar wind and then is forced back up in the sunward direction through the central plasma sheet region (Axford and Hines, 1961). The detail variation due to flow motion within and around those vortices is described in Figure $5 \mathrm{a}$ as it would be expected at four different distances from the magnetopause and that are represented by the dash lines A, B, C, and D. For a tailward moving vortex pattern (indicated by changes in the arrows of the flow direction) a waving motion should be apparent along the path line A which is located 
closer to the center of the tail. While along the path line B there should be a complete clockwise rotation (measured from the bottom to the top) a wavy periodic reversal will occur again along the path line $\mathrm{C}$, and a counterclockwise rotation will prevail along the path line D. These variations serve to describe the structure of the vortices as seen in the earth's rest frame. On the other hand, the flow pattern in the rest frame of the tailward moving wave is sketched in Figure $5 b$ to show how the shape of the vortex structure is maintained within that wave (Hones et al., 1981).

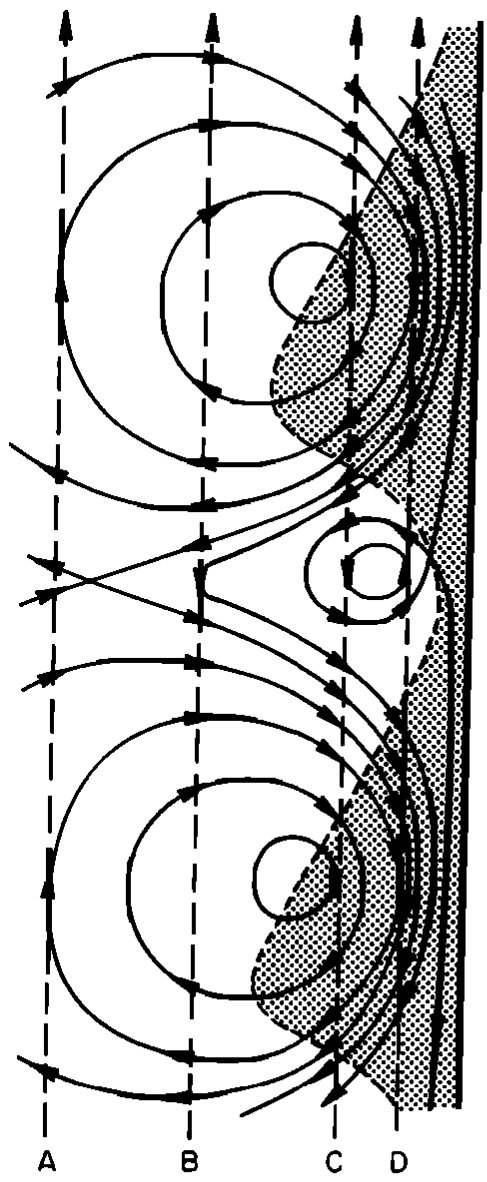

(a)



(b)

Figure 5. - (left panel) Dawn sector of the flow pattern in Figure 4 depicting the sequence of flow vectors observed at four different distances from the magnetopause. (right panel) Flow in the rest frame of the tailward moving wave (Hones et al., 1981). 
The wavy pattern in the velocity direction that is implied from the measurements reproduced in Figures 2 and 4 could result from effects associated with Kelvin-Helmholtz instabilities produced along the boundary of the magnetosphere. Such structures require, however, fluid dynamic processes produced under conditions in which the flow is superalfvenic; namely, that the local value of the kinetic energy density of the plasma is larger than the local magnetic energy density. As a whole this peculiarity is not applicable to the plasma that pervades within the inner magnetosphere where the magnetic energy density is far larger than the local kinetic energy density. However, different conditions occur along the tail where the magnetic field intensity gradually decreases with the downstream distance from the earth thus reducing the ratio of the magnetic energy density to the kinetic energy density. A suitable comparative calculation of the conditions that were present at the time when the plasma vortices of the Cluster data of Figure 1 were identified can be conducted by using the corresponding pressure profiles of those data and that are reproduced in Figure 6. Notable is that the plasma vortices in Figure 1 were detected after the dynamic pressure of the solar wind exhibited a sudden increase indicated by the dashed vertical line at 12:55 UT in Figure 6. The dynamic pressure $\mathrm{P}_{\mathrm{D}} \sim 3 \mathrm{nPa}$ of the solar wind after that event shown in the upper panel is over 10 times larger than the $\sim 0.16 \mathrm{nPa}$ magnetic pressure that can be derived from the $\sim 20 \mathrm{nT}$ magnetic field intensity values measured at the same time and that are shown in the third panel. The implication here is that the local flow is superalfvenic thus suggesting conditions that could have led to Kelvin-Helmholtz waves along the magnetopause.

\section{Plasma vortices in the Venus wake (PVO measurements)}

Measurements conducted within and along the flanks of the Venus wake have revealed conditions that are also suitable for the onset of fluid dynamic processes that lead to the generation of vortex structures. From observations made with the early Mariner 5 spacecraft it was noted that the solar wind that streams around Venus is subject to a large decrease of its momentum flux as its speed $U$ and density $n$ in the wake become significantly smaller than the values measured under freestream conditions (Bridge et al., 1967; Shefer et al., 1979). A summary of those measurements is presented in Figure 7 to show the presence of a velocity boundary layer that extends along the flanks of the Venus wake (indicated by the black shaded region) where both plasma properties exhibit strong deficient values. Such changes are bounded by a plasma transition labeled with the items 2 and 4 along the trajectory of the spacecraft in the lower panel and also at the top of the upper panel, being different from the Venus bow shock encountered at the items labeled 1 and 5 (in the latter case the plasma density is larger in the downstream side as a result of the compressed density values that the solar wind acquires across the bow shock). Much of the missing amount of momentum flux $\left(\mathrm{nU}^{2}\right)$ of the solar wind in the wake that is implied from the profiles in Figure 7 between items 2 and 4 has been found 


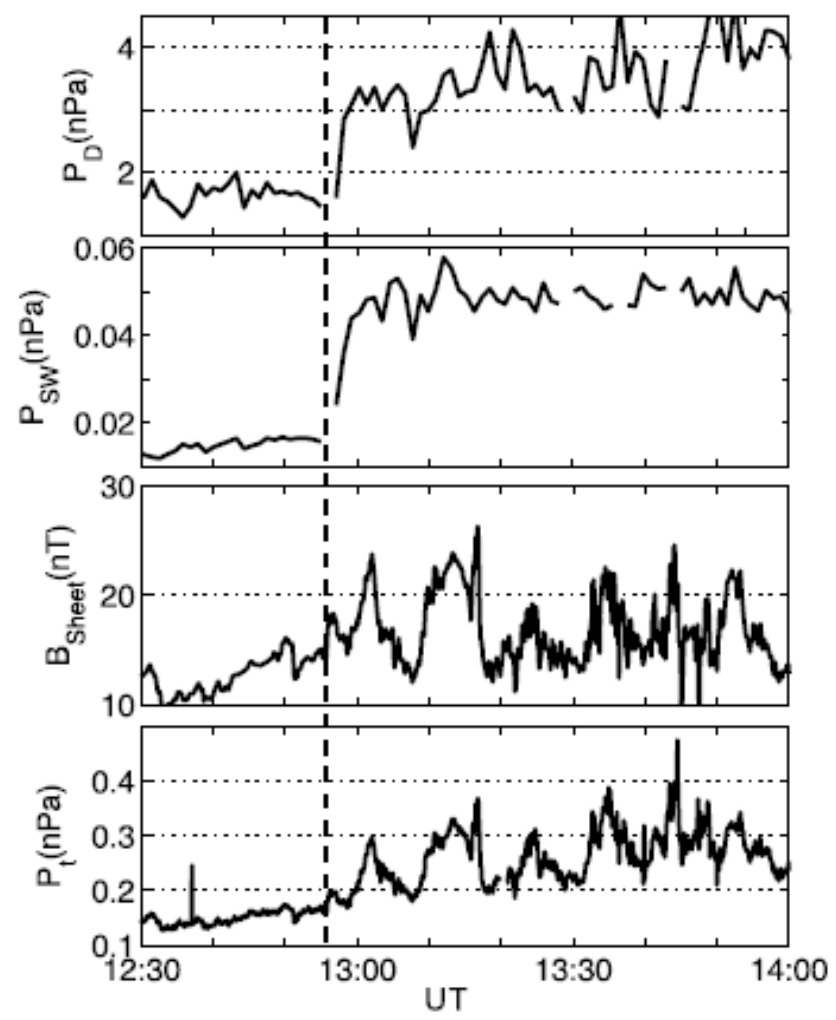

Figure 6. - Pressure variations of the solar wind dynamic pressure (top panel) and its static pressure (second panel), together with the magnetic field intensity (third panel) and the plasma pressure (bottom panel) of the plasma sheet that were measured on July 6, 2003 with a Cluster spacecraft (Figure 12 in Tian et al., 2010).

to be comparable to the momentum flux of the plasma that streams in the trans-terminator flow within the Venus upper ionosphere (Perez-de-Tejada, 1986) and that was measured with the Pioneer Venus Orbiter (PVO) spacecraft (Knudsen et al: 1980). The implication of this agreement is that there is an efficient transport of solar wind momentum to the Venus upper ionosphere that could be accounted for through the onset of viscous forces in a continuum fluid interpretation. As it was indicated before such processes require small scale interactions among the particles of both populations in view that the solar wind is a collisionless plasma. Wave-particle interactions resulting from the turbulence associated with strong fluctuations in the magnetic field that are measured along the flanks of the wake (Bridge et al., 1967; Vörös et al., 2008) should be ultimately responsible for the erosion of the ionospheric particles produced by the solar wind and, at the same time, for the the Kelvin-Helmholtz instabilities that develop under the measured superalfvenic flow conditions (Pèrez-de-Tejada et al., 2011). 

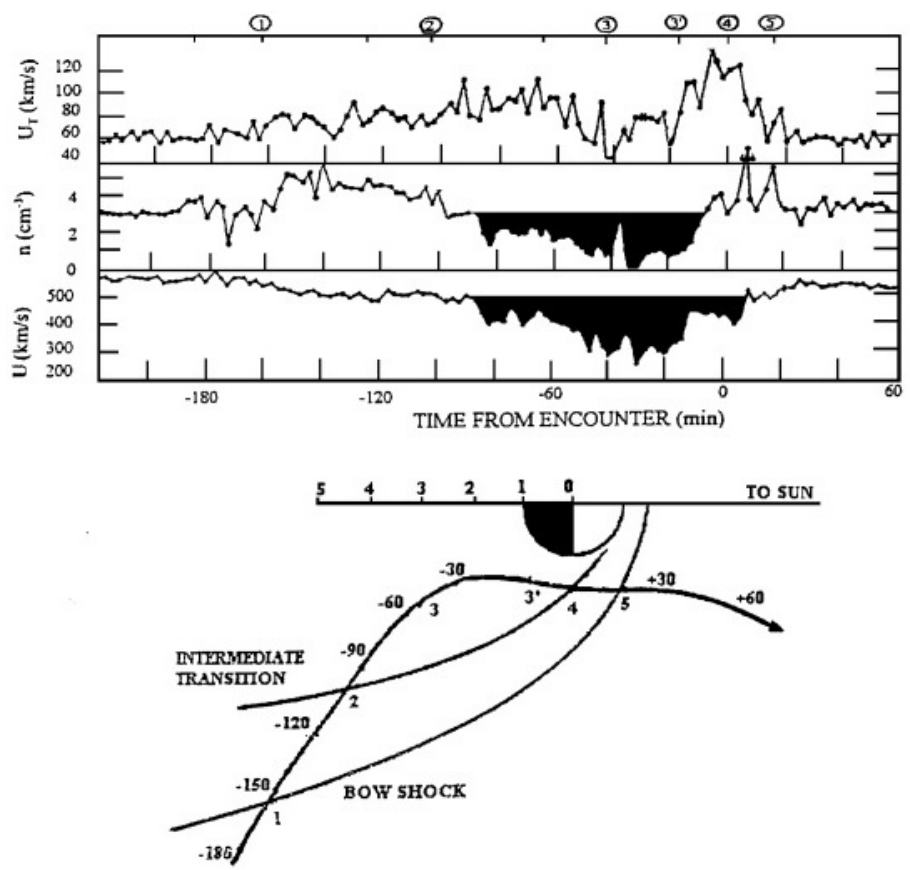

Figure 7. - Thermal speed, density, and bulk speed of the solar wind measured with the Mariner 5 spacecraft (its trajectory projected in cylindrical coordinates is shown in the lower panel). The labels 1 through 5 along the trajectory and at the top of the upper panel mark important events in the plasma properties (bow shock, intermediate plasma transition) (after Bridge et al..1967).

The presence of a velocity boundary layer along the flanks of the Venus wake has been confirmed with observations conducted with the PVO and more recently with the Venus Express (VEX) spacecraft (Pérez-de-Tejada et al., 2011). In the data obtained with both vehicles it has been possible to identify features within that layer that can be interpreted in terms of fluid dynamic processes and, in particular, vortical structures with properties similar to those detected in the earth's magnetosphere. The first indication on the existence of plasma vortices in the Venus wake was inferred from the observation of changes in the velocity direction of plasma particles along the PVO trajectory across the Venus wake (Pérez-de-Tejada et al., 1982). In some instances the ion fluxes are not directed away from the sun but their motion has a sunward directed component. A description of such change serves to identify the manner in which the flow is arranged to form vortical structures and their position within the wake.

From the collection of plasma ion fluxes measured during the first seasons of observation of the PVO we will first refer to a set of plasma data in which the velocity vectors of the ion fluxes exhibit sudden changes in the direction of the particle motion. Those data are presented in Table I and apply to PVO measurements conducted in orbits 80, 68, 65 and 66, which were conducted in the vicinity of the midnight plane during the first season of observation 
of that spacecraft along its nearly polar oriented trajectory. The measurements conducted in the energy cycle for each orbit correspond to those obtained near the outbound (southern hemisphere) crossing of the upper boundary of the ionosphere (ionopause) and represent the most intense ion fluxes that were observed during that cycle. Each reading in the first and second columns describes the time (in UT) when it was made together with the number of the corresponding energy step and the volt per charge value (in parenthesis). The count number of the flux intensity (which leads to the intensity values given in the right side column) and the latitudinal collector of the plasma instrument where it was received (marked by the number 3 or 4 ) are indicated in the third column. These values together with the calculated particle speed if they represent either $\mathrm{H}+$ or $\mathrm{O}+$ ions, and the azimuthal sector (and also the azimuthal angle $\alpha$ ), are presented in the fourth through the seventh columns. The most notable example in the data for orbit 80 shown at the top are measurements in which there are fluxes with a sunward directed component (negative $\alpha$ values). These are observed between the energy steps number 11 and 16 (first column), and there are also ion fluxes measured along the solar wind direction (positive $\alpha$ values) between the energy steps number 17 and 21. Comparable variations are also present in the data set of the other orbits with a distribution of the azimuthal $\alpha$ angle that is persistent in orbit 68 (as in orbit 80 ), and then fluctuating orientations in orbits 65 and 66.

A schematic representation of the direction of the particle fluxes in orbit 80 is described in the upper panel of Figure 8 for 3 different energy cycles of measurements together with the PVO trajectory projected on one quadrant using cylindrical coordinates (the outbound pass with cycles II and III occurs in the southern hemisphere but has been projected to the same quadrant of the inbound pass of cycle I that occurs in the northern hemisphere). The position of the spacecraft during the energy cycle I initiated at 19:34.27 UT in the inbound pass, and the energy cycles II and III initiated at 19:59.16 UT and at 20:08.43 UT in the outbound pass are shown in that figure (each cycle is marked by a rectangular shape). The arrows indicate the latitudinal direction of arrival of the particle fluxes corresponding to the orientation of the collector of the plasma instrument where they were observed (measurements made in collector labeled 4 in Table I during the outbound pass correspond to particle fluxes reaching the most northbound direction detected by the instrument $\left(22.5^{\circ}<\theta<69^{\circ}\right)$ and is opposite to those made in collector 1 which would be the most southbound directed collector of the instrument [Intriligator et al., 1980]. During the energy cycle I in the inbound pass and also in the energy cycle III in the outbound pass there is a tendency for the particle fluxes to be directed away from the wake, implying the observation of northbound fluxes in the northern hemisphere (collectors 3 and 4) and also the observation of southbound fluxes in the southern hemisphere (collectors 1 and 2). Different conditions can be identified in the energy cycle II measured in the vicinity of the outbound crossing of the ionopause that is reported in Table I. In this cycle the (dominant) particle fluxes now converge toward the wake (all are detected in collectors 3 or 4 ) and exhibit directions either with a sunward component (negative $\alpha$ values) or with an anti-sunward component (positive $\alpha$ values). This later variation is indicated by large differences in the azimuthal sector number of the measurements which implies angles that may differ by up to $180^{\circ}$ between both cases $(\alpha=0$ corresponding to the antisolar direction). 
Orbit 80 (19:59.16 UT)

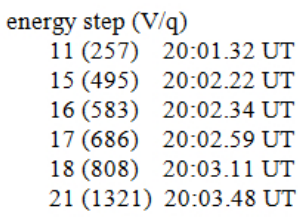

Orbit 68 (20:16.59 UT)

\begin{tabular}{rrrrrc} 
count & \multicolumn{2}{c}{ speed $(\mathrm{km} / \mathrm{s})$} & sector & $\alpha\left({ }^{\circ}\right)$ & ions $/ \mathrm{cm}^{2} \mathrm{~s} \mathrm{sr}$ \\
$107-4$ & 236 & 59 & 119 & -161 & $3.710^{7}$ \\
$101-4$ & 308 & 77 & 109 & -168 & $3.210^{7}$ \\
$101-3$ & 336 & 84 & 101 & -173 & $3.210^{7}$ \\
$95-4$ & 360 & 90 & 367 & 14 & $2.810^{7}$ \\
$106-4$ & 392 & 98 & 369 & 15 & $3.610^{7}$ \\
$101-4$ & 504 & 126 & 375 & 19 & $3.510^{7}$
\end{tabular}

\begin{tabular}{rccccc} 
count & \multicolumn{2}{c}{ speed $(\mathrm{km} / \mathrm{s})$} & sector & $\alpha\left({ }^{\circ}\right)$ & ions $/ \mathrm{cm}^{2} \mathrm{~s} \mathrm{sr}$ \\
$102-3$ & 96 & 24 & 438 & 65 & $3.310^{7}$ \\
$104-4$ & 104 & 26 & 430 & 58 & $3.510^{7}$ \\
$107-4$ & 124 & 31 & 192 & -109 & $3.710^{7}$ \\
$96-4$ & 284 & 71 & 195 & -107 & $2.910^{7}$ \\
$97-4$ & 308 & 77 & 190 & -111 & $2.910^{7}$ \\
$98-3$ & 336 & 84 & 200 & -104 & $3.010^{7}$
\end{tabular}

Orbit 65 (20:16.39 UT)

$\begin{array}{cc}\text { energy step no. (V/q) } \\ 2(59) & 20: 16.51 \text { UT } \\ 3(69) & 20: 17.04 \text { UT } \\ 4(82) & 20: 17.16 \text { UT } \\ 10(218) & 20: 19.03 \text { UT } \\ 15(495) & 20: 19.45 \text { UT } \\ 18(808) & 20: 20.35 \text { UT } \\ 21(1122) & 20: 21.12 \text { UT } \\ 25(2543) & 20: 22.14 \text { UT } \\ 26(2996) & 20: 22.26 \text { UT }\end{array}$

Orbit 66 (20:19.25 UT)

\begin{tabular}{rcc} 
count & \multicolumn{3}{c}{ speed $(\mathrm{km} / \mathrm{s})$} \\
$105-4$ & 104 & 26 \\
$99-4$ & 116 & 29 \\
$107-4$ & 124 & 31 \\
$101-4$ & 208 & 52 \\
$113-4$ & 308 & 77 \\
$98-4$ & 392 & 98 \\
$100-4$ & 504 & 126 \\
$100-4$ & 700 & 175 \\
$100-4$ & 732 & 183
\end{tabular}

sector
435
437
176
185
176
439
189
449
195

$\alpha\left({ }^{\circ}\right)$
6
63
-120
-114
-120
65
-111
71
107

ions $/ \mathrm{cm}^{2} \mathrm{~s} \mathrm{sr}$

$3.510^{7}$

$3.110^{7}$

$3.710^{7}$

$3.210^{7}$

$4.210^{7}$

$3.010^{7}$

$3.210^{7}$

$3.210^{7}$

$3.210^{7}$

\begin{tabular}{ccccr} 
energy step (V/q) & & count & \multicolumn{2}{c}{ speed $(\mathrm{km} / \mathrm{s})$} \\
$1(50)$ & $20: 19.25$ UT & $107-4$ & 96 & 24 \\
$9(185)$ & $20: 21.16$ UT & $116-4$ & 188 & 47 \\
$11(257)$ & $20: 21.41$ UT & $102-4$ & 220 & 55 \\
$14(420)$ & $20: 22.18$ UT & $109-4$ & 284 & 71 \\
$17(656)$ & $20: 23.08$ UT & $117-4$ & 364 & 91 \\
$24(2159)$ & $20: 24.35$ UT & $100-4$ & 644 & 161
\end{tabular}

sector
182
449
200
127
456
350

$\alpha\left({ }^{\circ}\right)$
-11
71
-104
-155
76
2

ions $/ \mathrm{cm}^{2} \mathrm{~s}$ sr

$3.110^{7}$

$4.610^{7}$

$3.310^{7}$

$3.910^{7}$

$4.710^{7}$

$3.210^{7}$

Table 1. Table I: Values of the ion flux intensity (right side column) measured during an energy cycle in the outbound pass of the VO in orbits $80,68,65$, and 66 , through the Venus near wake. The time when the ion fluxes are measured are given in the second column, and the corresponding energy step number with its volts per charge value (in parenthesis) are presented in the first column. Their count number and the latitudinal collector of the plasma instrument where the fluxes were measured is shown in the third column (fluxes in collectors 3 and 4 are northbounddirected implying that fluxes converge toward the plasma wake in the southern (outbound measured) hemisphere). Their speed for $\mathrm{H}+$ and $\mathrm{O}+$ ions is indicated in the fourth and fifth columns. The azimuthal sector and the corresponding azimuthal angle a where the fluxes were detected are indicated in the sixth and seventh columns (sectors above and below 347.5 correspond to positive and negative a values where in the later case it implies sunward directed fluxes).

A similar distribution of velocity vectors in the Venus wake is observed in the data of orbit 68 of Table I in energy cycles whose position along the PVO trajectory is reproduced in the lower panel of Figure 8. As it was the case for orbit 80 in the upper panel of that figure the 
northbound directed diverging particle fluxes are measured in the inbound pass (energy cycle I initiated at 19:53.22 UT), and southbound directed fluxes that also diverge from the wake are observed in the energy cycle III of the outbound pass initiated at 20:26.12 UT.

In addition, particle fluxes measured in the inner ionosheath during cycle II in the outbound pass (initiated at 20:16.59 UT) and that were included in Table I, converge toward the wake and like those in orbit 80 also include velocity vectors with a sunward directed component (most notable flux intensities in the $4-16$ energy steps) but there are also sporadic weak fluxes with an anti-sunward directed component in the lowest energies that were measured. The tendency in the data set of the outbound pass of orbits 80 and 68 is that the solar wind streaming near the ionopause (cycle labeled II in Figure 8) can acquire an orientation with a sunward directed component leading to a vortex structure $\left(\sim 180^{\circ}\right.$ change of the azimuthal $\alpha$ angle in that region).
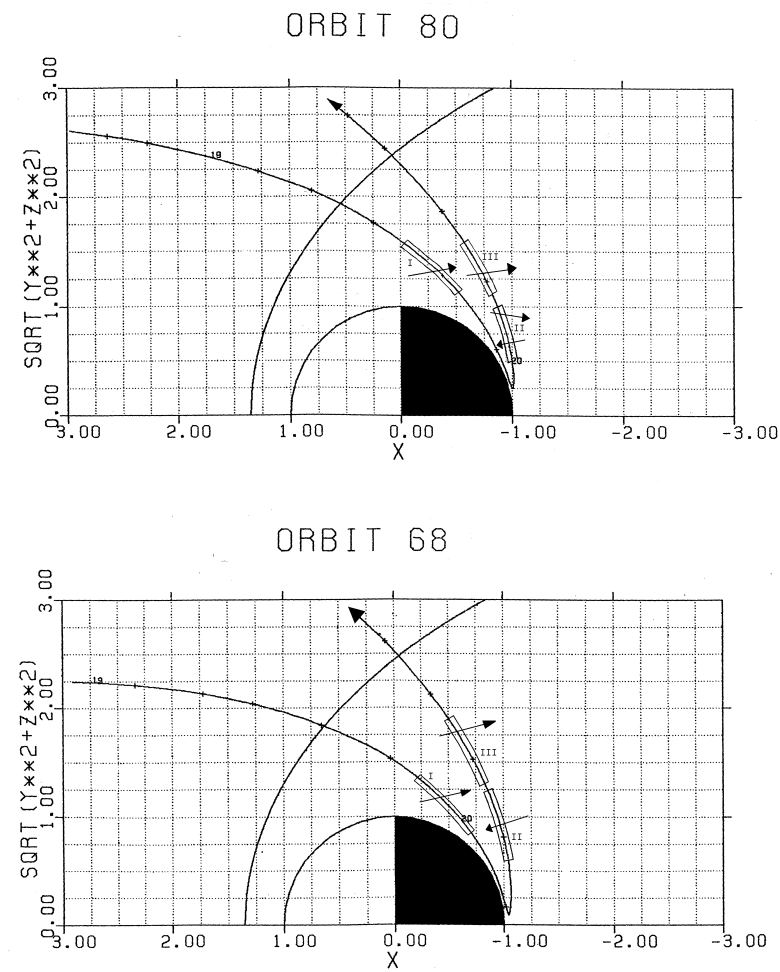

Figure 8. - Representative position of energy cycles (rectangular shapes) where measurements were made along the trajectory of the PVO in orbit 80 (upper panel) and in orbit 68 (lower panel) projected on a quadrant in cylindrical coordinates. The arrows show schematically the (latitudinal) velocity direction of ion fluxes detected at different energy steps within each cycle. 


\section{Plasma vortices in the Venus wake (VEX measurements)}

More conclusive evidence in the observation of sunward directed plasma fluxes in the Venus wake has been recently reported from measurements conducted with the ASPERA-4 instrument of the Venus Express spacecraft [Lundin et al., 2011]. From a collection of velocity vectors derived from measurements conducted in 380 orbits it was possible to produce the average pattern of $\mathrm{O}+$ and solar wind $(\mathrm{SW}) \mathrm{H}+$ ion velocity vectors presented in Figure 9 which are projected in cylindrical coordinates. Notable is that most of the velocity vectors of the $\mathrm{O}+$ ions are deviated towards the inner wake. On the other hand, for the majority of the solar wind $\mathrm{H}+$ ions the velocity vectors by the central tail at $\mathrm{x}<-2 \mathrm{R}_{\mathrm{V}}$ have a sunward directed component. Such variation mostly occurs in the central wake but in the solar wind $\mathrm{H}+$ ion panel there is also evidence of that behavior in the velocity vectors located downstream from the polar region. The overall pattern of the velocity vectors is consistent with plasma fluxes that reverse direction and agrees with a similar structure that was inferred from the PVO observations; that is, velocity vectors with a sunward component and some directed away from the wake can be separately identified in Figure 9, thus indicating the presence of divergent and sunward directed ion fluxes as it was inferred from the PVO data.
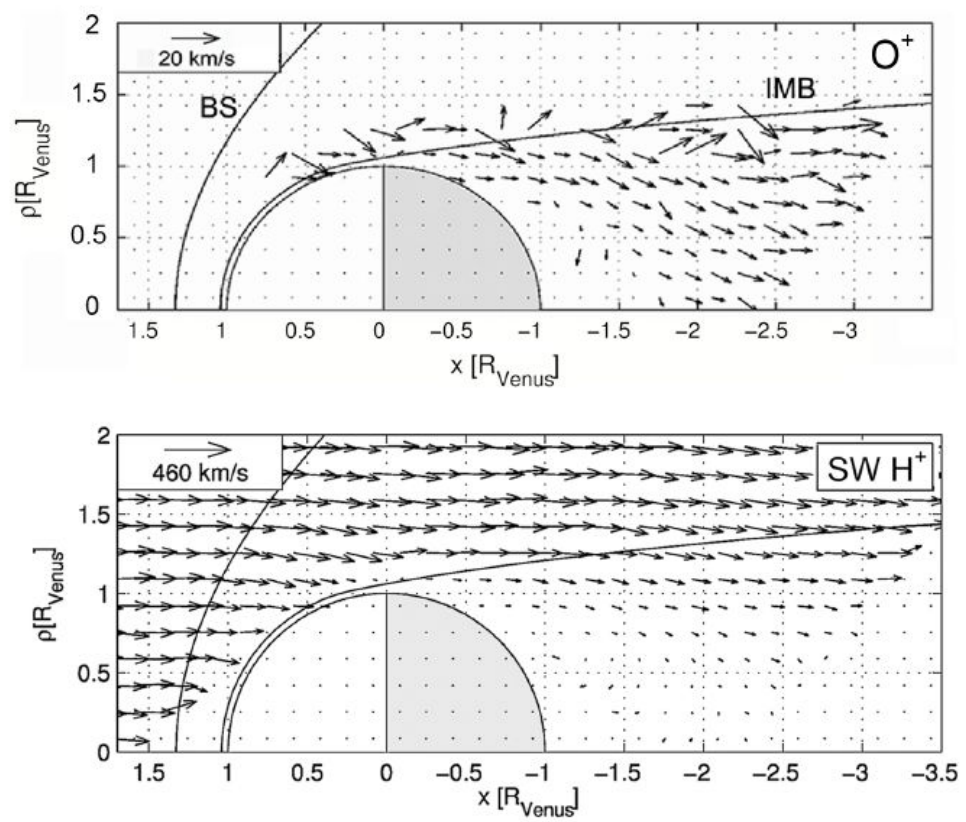

Figure 9. - Velocity vectors of out-flowing $\mathrm{O}+$ (upper panel) and solar wind $\mathrm{H}+$ (lower panel) ions measured in the Venus wake with the ASPERA-4 instrument of the Venus Express spacecraft projected in cylindrical coordinates. The velocity scales for the $\mathrm{O}+$ and the $\mathrm{H}+$ ions are noted in the upper left corner in each diagram. Notice that the $\mathrm{H}+\mathrm{flow}$ velocities in the wake are low and barely discernible. However, applying unit vectors we find that most solar wind $\mathrm{H+}$ velocity vectors are sunward directed near the tail central plane at $x<2 R_{V}$. 
A more detail description of the distribution of velocity vectors measured with the ASPERA-4 instrument of the VEX spacecraft is presented in Figure 10 with an account of separate observations of the solar wind $\mathrm{H}+$ and the $\mathrm{O}+$ ion fluxes with values now averaged along the $\mathrm{z}$-direction and subsequently projected to the xy plane. Most notable in the left panel of Figure 10 is a pattern located in the central wake within which the velocity vectors of the $\mathrm{H}+$ ions in the dusk side (negative y-values) show a common tendency to be deflected towards the dawn side (positive $y$-values), and also vectors located at and in the vicinity of the midnight meridian that exhibit a strong solar oriented component. This pattern is consistent with the onset of reverse flow conditions that seem to occur slightly shifted towards the dawn side. As a whole the velocity vectors in that region contain cases in which they are directed anti-sunward, some are deflected toward positive values in the $y$-axis, and in various observations they are mostly sunward oriented. A scheme with different properties is observed in the distribution of velocity vectors of the planetary $\mathrm{O}+$ ion population shown in the right panel of Figure 10. In this case the velocity vectors across the wake show a more thorough deflection toward the dawn side (positive $y$-values) but there is little or no indication in the xy-projection (values averaged along the $\mathrm{z}$-axis) that they have a sunward directed component.
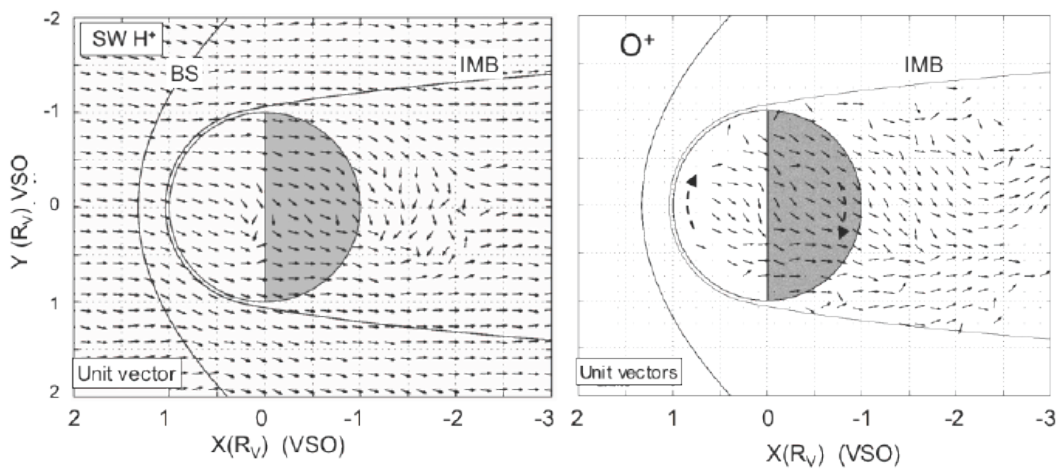

Figure 10. - (left panel) Distribution of velocity vectors of the solar wind $\mathrm{H}+$ ion population in the Venus wake obtained with the ASPERA-4 instrument of the Venus Express projected on the xy plane (the data show evidence of a region in the central wake where the velocity vectors acquire a sunward directed component). (right panel) Distribution of velocity vectors of the $\mathrm{O}+$ ion population indicating that ions in the dusk (upper) side of the retrograde rotating Venus ionosphere are deviated toward the positive (dawn side) y-direction (Lundin et al., 2011).

From the sunward directed fluxes reported in the left panel of Figure 10 the reversed flow direction should mostly occur for the $\mathrm{H}+$ population and, as it is the case for obstacles immersed in directional flows, the solar wind particles in the wake will be forced in the upstream direction leading to a vortex structure. A schematic representation of the distribution of flow streamlines that is compatible with this view is indicated in the left panel of Figure 11 with a circulation pattern in the wake that is consistent with the plasma data. The rotation of the flow direction in the wake is supported by the diagram presented in the right panel of Figure 11 where a wide velocity boundary layer with a thickness that is larger downstream from the terminator stresses the low local flow speeds that should occur near the ionosphere. Such velocity layer derives from the observations shown in Figure 7 and is 
produced by viscous transport of solar wind momentum to the upper ionospheric plasma. Since low speeds are expected close to the ionosphere the solar wind will reach a position within the wake where the large local flow pressure values will produce a change in its direction thus leading to a vortex structure as that depicted for a flow past an obstacle indicated in the right panel of Figure 11. It is to be noted that the fluid dynamic response of the solar wind to the large pressure values present in the wake is different from processes associated with Kelvin-Helmholtz instabilities along the boundary of the ionosphere and that, as it is the case in the earth's magnetosphere, also contribute to produce vortex structures.
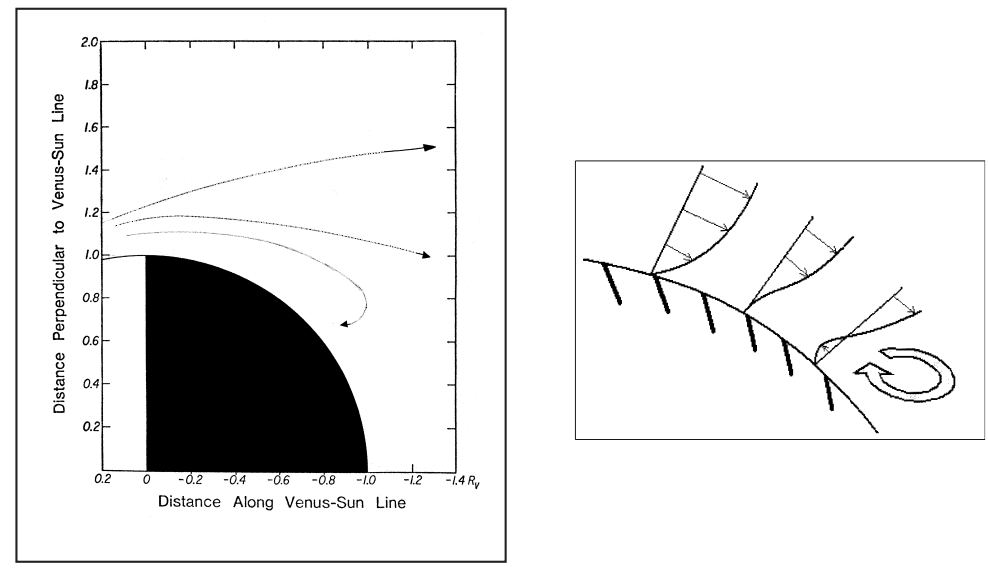

Figure 11. - (left panel) Schematic representation of a vortex structure generated downstream from a polar region of the Venus ionosphere (the streamlines indicate the direction of motion of the solar wind). (right panel) Flow pattern within a velocity boundary layer that extends past an obstacle (higher pressures in the wake reverse the flow direction near the obstacle leading to a vortex structure, Schlichting, 1968).

The fluid dynamic interpretation of both phenomena serves to account for the processes that produce the vortex structures in the earth's magnetosphere and in the Venus plasma wake, and should also be applicable to the conditions that are expected in other planets; namely, those with an appreciable or strong intrinsic magnetic field (Jupiter, Saturn, Uranus, Neptune, and Mercury), or in other un-magnetized planets in which there may only be fossil remnants of an early magnetic field as it is the case in Mars (Acuña et al., 1999).

\section{Author details}

H. Pérez-de-Tejada ${ }^{1^{*}}$, Rickard Lundin ${ }^{2}$ and D. S. Intriligator ${ }^{3}$

1 Institute of Geophysics, UNAM, México, México

2 Institute of Space Physics, Umea, Sweden

3 Carmel Research Center, Santa Mónica, California, USA 


\section{References}

[1] Acuña, M., et al. (1999). Global distribution of crustal magnetization discovered by the Mars Global Surveyor MAG/ER experiment. Science, 790 EOF.

[2] Axford, I., \& Hines, C. (1961). A unifying theory of high latitude geophysical phenomena and geomagnetic storms in Canada. Journal of Physics,.., 39, 1433 EOF-1464 EOF.

[3] Bridge, H., , A., Lazarus, C., Snyder, E., Smith, I., Davies, P., Coleman, , \& Jones, D. (1967). Plasma and magnetic fields observed near Venus,. Science, , 158, 1669.

[4] Hones Jr, E., et al. (1978). Vortices in magnetospheric plasma flow. Geophys. Res. Letters, , 5, 1059 EOF-1062 EOF.

[5] Hones Jr, E., et al. (1981). Further determination of the characteristics of magnetospheric plasma vortices with Isee 1 and 2. J. Geophys. Res.,, 86, 814 EOF-820 EOF.

[6] Hones Jr, E., et al. (1983). New observations of plasma vortices and insights into their interpretation. Geophys. Res. Letters,, 10, 674 EOF-677 EOF.

[7] Intriligator, D. J., Wolfe, , \& Mihalov, J. (1980). The Pioneer Venus Orbiter plasma analyzer experiment. IEEE Trans, Geosci. Remote Sensing,, GE-18, 39 EOF-43 EOF.

[8] Knudsen, W., et al. (1980). Transport of ionospheric $\mathrm{O}^{+}$ions across the Venus terminator and implications. J. Geophys. Res.,, 85, 7803 EOF-7810 EOF.

[9] Lundin, R. S., Barabash, Y., Futaana, J. A., Sauvaud, A., Fedorov, , \& Perez-de-Tejada, H. (2011). Ion flow and momentum transfer in the Venus plasma environment. ICARUS, 751 EOF-758 EOF.

[10] Pérez-de-Tejada, H., Gay-García, C., Intriligator, D., \& Dryer, M. (1982). Plasma vortex in the Venus near wake,. EOS, 63(18), 368.

[11] Pérez-de-Tejada, H. (1986). Fluid dynamic constraints of the Venus ionospheric flow. J. Geophys. Res.,, 91, 6765 EOF-6770 EOF.

[12] Pérez-de-Tejada, H., Lundin, R., Barabash, S., Zhang, T. L., Coates, A., Sauvaud, J. A., Durand-Manterolla, H., \& Reyes-Ruiz, M. (2011). Plasma transition along the flanks of the Venus ionosheath: Evidence from the Venus Express plasma data,. Journal of Geophys. Res.,, 116, A01103, doi: JA015216.

[13] Pérez-de-Tejada, H. (2012). Fluid dynamics in space sciences,. chapter 28, 611, Fluid dynamics, computational modeling and applications," Hector Juarez, Ed., INTECH,, 978-9-53510-052-2, open access.

[14] Pope, S., et al. (2009). Giant vortices lead to ion escape from Venus and redistribution of plasma in the ionosphere,. Geophys. Res. Lett., 36, L07202.

[15] Schlichting, H. (1968). Boundary Layer Theory,. McGraw Hill, 408. 
[16] Shefer, R., Lazarus, A., \& Bridge, H. (1979). A re-examination of plasma measurements from the Mariner 5 Venus encounter,. J. Geophys. Res.,, 84, 2109.

[17] Tian, A., et al. (2010). A series of plasma flow vortices in the tail plasma sheet associated with solar wind pressure enhancement. J. Geophys. Res., 115, A09204, doi: JA014989.

[18] Terada, N., Machida, S., \& Shinagawa, H. (2002). Global hybrid simulation of the Kelvin-Helmholtz instability at the Venus ionopause,. J. Geophys. Res.,, 107, 14.

[19] Wolfe, R., et al. (1980). The onset and development of Kelvin-Helmholtz instability at the Venus ionopause,. Journal of Geophys. Res.,, 85, 7697-7707.

[20] Vőrős, Z., et al. (2008). Intermittent turbulence, noisy fluctuations, and wavy structures in the Venusian magnetosheath and wake,. Journal of. Geophys. Res.-Planets,, 113, E00B21, doi:10.1029/2008. 